\title{
Performance of a Diamond-Tungsten Sampling Calorimeter
}

R. J. Tesarek, R. Gilman, G. Kumbartski, M. H. Lee, ${ }^{*}$ P. Rutt, F. Sannes, S. Schnetzer, S. V. Somalwar, R. Stone, G. B. Thomson

Department of Physics and Astronomy

Rutgers University, Piscataway, NJ 08855-084.9

\author{
M. Franklin \\ Physics Department \\ Harvard University, Boston, MA 02138 \\ S. Kanda \\ Department of Physics \\ University of Hawaii, Honolulu, HI 96822 \\ B. Morozov, ${ }^{\dagger}$ Y. Sugimoto \\ KEK National Laboratory \\ Tsukuba-shi, Ibaraki-ken 305, Japan \\ S. $\operatorname{Han}^{\ddagger}$, D. R. Kania, L. S. Pan \\ Laser Division \\ Lawrence Livermore National Laboratory \\ Livermore, CA 94550
}

D. Fujino, K. K. Gan, J. Hassard ${ }^{\S}$, H. Kagan, R. Kass, R. Malchow, ${ }^{* *}$ S. Margetides, ${ }^{\S}$ W. Palmer, C. White, S. Zhao

Department of Physics

The Ohio State University, Columbus, OH 43210

\author{
S. K. Kim \\ Physics Department \\ Seoul National University, Seoul 151-742, Korea
}

(June 1, 1994)

\footnotetext{
*Present address: Physics Department, Seoul National University, Seoul, Korea 151-742

${ }^{\dagger}$ Present address: Joint Institute for Nuclear Research, Dubna, USSR.

$\ddagger$ Present address: Physics Research Division, LANL, Los Alamos, NM 87545

${ }^{\S}$ Permanent address: Imperial College, London, England SW72BZH

** Present address: Physics Department, Colorado State University, Ft. Collins, CO 80523
} 


\begin{abstract}
We report here the first measurements of a diamond-tungsten sampling calorimeter. The calorimeter consisted of twenty layers of diamond with one radiation length of tungsten per layer. The diamond layers were grown by chemical vapor deposition and were $3.0 \times 3.0 \mathrm{~cm}^{2}$ wafers with an average thickness of $500 \mu \mathrm{m}$. We measured the energy response and resolution $\left(\sigma_{E} / E\right)$ of this calorimeter in 0.5 to $5.0 \mathrm{GeV}$ electron beams and compared the results with those from a silicon calorimeter of similar construction. Our energy resolution is $\sigma_{E} / E=(4.7 \pm 2.7) \% / E \oplus(19.13 \pm 0.86) \% / \sqrt{E} \oplus(2.3 \pm 1.8) \%$ for the diamond-tungsten calorimeter, where $\oplus$ indicates addition in quadrature. This is in good agreement with our result for the silicon-tungsten calorimeter of $\sigma_{E} / E=(3.89 \pm 0.87) \% / E \oplus(19.73 \pm 0.19) \% / \sqrt{E} \oplus(0.0 \pm 1.6) \%$. We also compare our data with EGS simulations.
\end{abstract}

Typeset using REVTEX 


\section{INTRODUCTION}

Future high luminosity hadron colliders will require high performance detectors with fast rise and recovery times that can survive in an extreme radiation environment. Detectors based on diamond hold great promise for this application. Diamond is a large bandgap $(5.5 \mathrm{eV})$ material, is radiation hard and has a very fast collection time, 2 ns over $300 \mu \mathrm{m}$ [1].

Polycrystalline diamond is currently grown in large area wafers by the method of chemical vapor deposition (CVD). The raw materials for this process consist of hydrogen and methane gases making the growth process potentially inexpensive. Industry presently uses this process to produce diamond heat sinks, optical windows and tool coatings. A program has also begun to develop high quality diamond for electronics. The diamonds for detectors in this test were produced using a modification of present industrial techniques.

The principle of operation of a diamond as an ionizing radiation detector is shown in Figure 1. An electric field is applied across the diamond. A charged particle passing through the diamond ionizes the material and the separation of the charges due to the applied field induces a signal on the surface electrodes. Since diamond is an excellent insulator, the leakage current is negligible even for fields $>10^{4} \mathrm{~V} / \mathrm{cm}$. As a result, the fabrication of a device is very simple with no need for a reverse biased $p-n$ junction. We present here the first measurements of the performance of a calorimeter using diamond detectors based on the principles described above. These measurements are compared with our own high statistics measurements of a silicon-tungsten calorimeter of similar configuration.

\section{EXPERIMENTAL DESIGN}

The calorimeter used in the tests presented here consisted of twenty layers of alternating $1.0 X_{0}$ thick pieces of tungsten and detector planes. Each detector plane was made of either a diamond or a silicon assembly mounted on a $1.6 \mathrm{~mm}$ thick G-10 PC board with associated preamplifier electronics. The active area of each detector assembly measured $3.0 \times 3.0 \mathrm{~cm}^{2}$ and was segmented into three channels defined by $1.0 \times 3.0 \mathrm{~cm}^{2}$ strips. The strips in alternate detector layers were oriented perpendicular to each other to provide shower position information in two dimensions. Slotted teflon spacers held the adjacent G-10 boards $6.4 \mathrm{~mm}$ apart. The spacers also held a $4.05 \times 4.05 \mathrm{~cm}^{2}$ piece of tungsten glued onto a $0.8 \mathrm{~mm}$ thick G-10 board centered in front of each detector plane. The design of the spacers allowed for the easy removal of the tungsten during calibration. Two additional planes of silicon were mounted in front of the calorimeter with their strips oriented perpendicular to each other. These two planes were used to align the calorimeter in the beam and to provide coarse beam position information. The twenty layers of the calorimeter and the two beam position layers were held together by four threaded stainless steel rods and enclosed in an aluminum box.

The diamond detectors were grown approximately $500 \mu \mathrm{m}$ thick on four inch discs using the CVD process by Norton Diamond Films, Inc. [2] and laser cut into $3.0 \times 3.0 \mathrm{~cm}^{2}$ squares. Each square was then plated on the substrate side with a single $2.90 \times 2.90 \mathrm{~cm}^{2}$ titanium-platinum-gold electrode [3] and on the growth side with three $0.93 \times 2.90 \mathrm{~cm}^{2}$ electrodes. A conductive glue [4] held the diamond detector by the substrate-side electrode to a $3.60 \times 4.70 \mathrm{~cm}^{2}$ by $400 \mu \mathrm{m}$ thick ceramic board. The three electrodes on the growth side 
of the diamond were wire bonded to gold traces on the ceramic board. Figure 2 shows the diamond detector assembly. Each diamond detector assembly was taped and electrically connected to a pre-amplifier board. Black plastic was taped over the detector assembly to keep out light.

The quality and operating voltage for each diamond detector was determined by measuring the bulk charge collection distance $\left(d_{c}\right)$, using a ${ }^{90} S r$ beta source [3,1,5], as a function of the voltage applied across the diamond. The charge collection distance is the average distance that an electron-hole pair separate. Collection distance data from a typical detector are shown in Figure 3. The collection distance was seen to vary no more than $10 \%$ across a single $3.0 \times 3.0 \mathrm{~cm}^{2}$ wafer. Table I contains a list of the operating voltages and collection distances of each of the diamond detectors and where they resided in the calorimeter.

The silicon detectors were manufactured by the Hamamatsu Corporation [6] and consisted of six $1.0 \times 1.5 \mathrm{~cm}^{2}$ photodiodes on a single $300 \mu \mathrm{m}$ thick silicon wafer which was mounted to a $400 \mu \mathrm{m}$ thick ceramic board. Pairs of photodiodes were ganged together forming a single electronic channel with an area of $1.0 \times 3.0 \mathrm{~cm}^{2}$. The silicon detector assemblies were attached to the pre-amplifier boards in the same manner as the diamond detectors. The silicon photodiodes were reverse biased at 70 volts which assured the silicon would operate at full depletion.

The diamond and silicon detectors were coupled to charge-sensitive preamplifiers by 2200 pf and 100 pf capacitors, respectively. The lower value for the silicon detector coupling capacitor was used to avoid saturation of the preamplifier and enabled us to use the same electronics for both the diamond and the silicon detectors. DIGITEX 1576, charge sensitive preamplifiers [7], with a gain of $30 \mathrm{mV} / \mathrm{fC}$, converted the collected charge into a voltage signal. Both diamond and silicon signals were attenuated and sent to a receiver/shaper circuit [8] with a $2.7 \mu \mathrm{s}$ peaking time. The resulting pulses were then digitized in a LeCroy $1885 \mathrm{~F}$ Fastbus ADC [9]. The ADC was operated in its auto-ranging mode to give good resolution for small pulses and to provide a large dynamic range. A $1.0 \mu s$ gate strobed the ADC at the peak of the receiver/shaper signal. Figure 4 is a schematic diagram of the electronics.

Three triggers were used, one beam trigger and two monitoring triggers, which will be referred to as pedestal and pulser. All triggers were taken throughout the data running and initiated the digitization and readout of the whole apparatus. The beam trigger required the coincidence of two scintillation counters placed in the beam upstream of the apparatus. The pedestal trigger was driven by a pulse generator that initiated the calorimeter readout when there was no other activity in the detectors. Pedestal triggers were used to monitor the stability of the ADC pedestals. The pulser triggers originated in a manner similar to the pedestal triggers but included a TTL pulse which was sent to a computer controlled attenuator. The resulting, attenuated signal was then fanned out to 1 pf test capacitors coupled to each pre-amplifier channel of the calorimeter. The pulser triggers were used to help calibrate the calorimeter electronics and to monitor changes in the gain of the electronics for each calorimeter channel. Once a trigger initiated the readout sequence, all subsequent triggers were inhibited until the readout sequence was completed and the electronics reset.

The experiment was controlled and the data recorded through a personal computer running PC Spuds [10]. The data acquisition program provided monitoring capability in the form of histograms. More detailed monitoring was obtained by programs running in almost 
real-time on a separate workstation. A diagram of the electronics and data acquisition system is shown in Figure 5.

The data presented in this report were taken at the IT4 beam line at the TRISTAN accumulator ring at KEK. The $6.5 \mathrm{GeV}$ stored electron beam was scraped using a target inside the accumulator ring vacuum pipe. Bremsstrahlung photons from the scraped beam were converted into $e^{+} / e^{-}$pairs. The momentum of the tertiary, electron beam was selected by tuning the settings of a dipole magnet which swept electrons through the aperture of a collimator. The energy response of the calorimeter was measured by using six different beam momenta over the entire available range $0.5-5.0 \mathrm{GeV} / \mathrm{c}$. Beam rates varied with the beam momentum from approximately $60 \mathrm{~Hz}$ for the $0.5 \mathrm{GeV} / \mathrm{c}$ beam to less than $1 \mathrm{~Hz}$ for the $5.0 \mathrm{GeV} / \mathrm{c}$ beam. The beam momenta used and the fractional momentum resolution $\left(\sigma_{P} / P\right)$, determined from a previous experiment, are given in Table II. The uncertainty in the momentum spread was determined to be approximately $8 \%$ of the values presented and is reflected in the table.

\section{CALIBRATION AND MONITORING}

Separate procedures were used to calibrate the electronics and the detector elements of the calorimeter. First, the electronic gain and linearity were determined for each channel using the pulser system described in the previous section. Then, the absolute gain of individual detector elements was determined using minimum ionizing tracks from single electrons from the beam with the tungsten removed from the calorimeter. By tracking the constants determined via these two procedures one could distinguish between effects due to either the electronics or the detector elements. Both the diamond and silicon calorimeters were calibrated using the same technique.

The electronic gain and linearity for each channel were determined by simultaneously pulsing all the preamplifiers channels. Approximately 1000 events were taken at attenuation settings varying from 0 to $75 \mathrm{~dB}$ while recording the digitized pulse height in each channel. The attenuated pulser output was measured using a digital sampling oscilloscope. A total of twenty attenuation level settings were used with approximately half of the settings occurring in the low range of the ADC and half in the high range. Linear fits to the ADC count versus pulse height spectrum for both the high and low ranges provided two gains for each electronic channel. The maximum deviation from the linear fit in either range $(($ data - fit $) /$ fit $)$ was $0.95 \%$ for the diamond calorimeter and $0.24 \%$ for the silicon calorimeter.

Pedestals were obtained from pedestal triggers for each data run which lasted approximately two hours.

The individual gains of the detector elements were determined using $0.5 \mathrm{GeV}$ electrons, effectively minimum ionizing particles (MIP's), passing through the calorimeter with the tungsten removed. The strips in both views were calibrated separately. Initial gains for each channel were obtained by estimating the mean of the minimum ionizing signal for a representative channel. These gains served as starting points for the iterative procedure described below. Requiring that the front and back layers in the appropriate view had one effective minimum ionizing particle in the corresponding strip $\left(0.5<E_{\text {chan }}<1.5\right.$ MIP's $)$ and pedestals in the other strips $\left(E_{\text {chan }}<0.5\right.$ MIP's) produced a clean signal spectrum for a given channel. The above requirements guaranteed that only one particle hit the 
detector and reduced contamination of the signal due to the electron showering in the PC boards on which the diamond or silicon was mounted. In addition, the same requirements were made for the layers of the corresponding view immediately in front and behind the layer of interest. These stricter requirements further cut down on any contamination due to showering upstream of the channel of interest. A relative channel gain (relative to the previous iteration) was calculated as the average of the signal spectrum over all events which satisfied the above criteria with the additional requirement that the channel signal be less than 4.0 MIP's ${ }^{1}$. A new channel gain was then determined by multiplying the gain from the previous iteration by the relative gain obtained above. This procedure was repeated until all the relative gains from the last iteration deviated from unity by less than $0.05 \%$ ( $0.05 \%$ was used only as a convergence criterion). Using this procedure, the gains of the diamond and silicon channels were determined to better than $2.4 \%$ and $1.7 \%$, respectively. The above measured signal was converted to an effective number of minimum ionizing tracks by dividing by the detector gain determined above.

Measuring the pedestal width in units of MIP's gives the signal/noise and the electronic noise for each channel. The average measured signal/noise for the two detector types was 2.1:1 for diamond and 26:1 for silicon. Signals from diamond detectors are intrinsically smaller than those for silicon due to the higher electron-hole (e-h) creation energy $(13.0 \mathrm{eV}$ for diamond versus $3.6 \mathrm{eV}$ for silicon). However, this is somewhat compensated by diamond's greater density $\left(3.5 \mathrm{~g} / \mathrm{cm}^{3}\right.$ for diamond, $2.3 \mathrm{~g} / \mathrm{cm}^{3}$ for silicon). Therefore, the mean energy loss of $0.5 \mathrm{GeV}$ electrons, as calculated from the restricted energy loss formula [11], implies 3600 and 7800 e-h pairs are created for each $100 \mu \mathrm{m}$ of diamond and silicon traversed, respectively. Measuring the pedestal width in units of MIP's gives the electronic noise for each channel. Combining the signal/noise information, the number of electrons produced and the collection distance of the diamond detectors gives an average electronic noise of 712 and 450 electrons for diamond and silicon, respectively. Figure 6 shows typical pedestals and signals (hatched regions) for both detectors.

ADC pedestals and electronic gains of all calorimeter channels were monitored throughout the silicon and diamond running using the pedestal and pulser triggers discussed in the previous section. Individual ADC pedestals were observed to vary $2.5 \mathrm{ADC}$ counts (0.01 MIP's) between runs in the diamond data sample. The pedestals for the silicon data sample varied 0.5 ADC counts (0.001 MIP's). The electronic gains were observed to change less than $1.0 \%$ for each of the two data samples.

\section{DATA ANALYSIS}

The response of the calorimeter for each event was determined by summing the pedestal subtracted and gain corrected signals from all the channels. By accepting events where only the center strips in the front two pieces of silicon were hit, a maximum accepted spot size of $1.0 \times 1.0 \mathrm{~cm}^{2}$ was defined and ensured that the shower was roughly centered in the calorimeter. As shown in Figure 8, there was a small constant background below the peak

\footnotetext{
${ }^{1}$ This cut was used initially to reduce the background due to showering in the calorimeter. A subsequent recalibration without this cut gives the same resolution as the calibration with the cut.
} 
of the calorimeter response spectrum. This background was attributed to scraping of the beam in the collimator upstream of the calorimeter. Therefore, in the final calculation of the mean and rms, events were rejected where the calorimeter response was more than three standard deviations below the mean. Estimates of the size of this background and its effect on the calorimeter resolution are discussed below. Both calorimeters were treated in the same manner except for the following corrections.

Two effects were observed in the diamond calorimeter which required corrections. First, one of the side strips in layer 16 (16 $X_{0}$ deep in the calorimeter) was very noisy. Therefore, the signal from this channel was replaced by the average of the signals in the corresponding strips in layers 14 and 18. Second, there was a systematic time variation of the diamond calorimeter gain in the first part of the electron calibration and in the $5 \mathrm{GeV}$ data that immediately followed. Figure 7 shows the normalized calorimeter response as a function of time after the diamond bias voltage was turned on for data taking ${ }^{2}$. The electronics was not responsible for this effect since, from pulser data, the electronic gain of each channel was found to be stable to better than $1 \%$. The solid line in the figure is a fit to a constant plus an exponential and yields a decay time of 380 minutes. The data were corrected for this time dependence resulting in a $3 \%$ shift in the mean calorimeter response at $5 \mathrm{GeV}$. The time dependent correction was negligible for data at other energies which were taken later. The effect of this time dependence on the energy resolution fit parameters was less than their uncertainties.

The $4 \mathrm{GeV}$ calorimeter response distributions are shown for both diamond and silicon in Figure 8. A summary of the corrected energy mean and rms in both detectors for all beam energies is found in Table II. The errors listed in the table are purely statistical.

A $1 \%$ systematic uncertainty in the central value of the beam energy is necessary to account for fluctuations in the linearity of both calorimeter responses. These fluctuations are consistent with hysteresis in the beam momentum selection magnet. This uncertainty is added in quadrature with the statistical uncertainties. Its effect on the uncertainty in the energy resolution was small compared with the statistical uncertainty. Figure 9 shows the diamond and silicon calorimeter responses as a function of beam energy. The errors shown include the uncertainty in the central value of the beam energy. The calorimeter response as a function of beam energy was then fit to a straight line giving the following results:

$$
\begin{aligned}
\epsilon_{\text {diamond }}\left(M I P^{\prime} S\right) & =(66.19 \pm 0.26) \cdot E_{\text {beam }} & \chi^{2}=10.84 / 5 \text { dof } \\
\epsilon_{\text {silicon }}\left(M I P^{\prime} s\right) & =(65.96 \pm 0.26) \cdot E_{\text {beam }} & \chi^{2}=8.35 / 5 \text { dof }
\end{aligned}
$$

As mentioned in the previous section, there was a small background due to the electron beam scraping the collimator. Again, the scraping background is constant below the peak in the calorimeter response distributions. The $3 \sigma$ lower end cut removes most of this background, but some small amount remains under the peak. This residual background is estimated assuming that it is constant from the cut value to the mean of each response spectrum and zero elsewhere. The rms of this background about the previously determined mean for this energy was then calculated. This rms was then scaled by the ratio of the esti-

\footnotetext{
${ }^{2}$ Prior to data taking, the diamond bias voltage was cycled on and off for approximately 8 hours to test the calorimeter and fix minor problems with the electronics.
} 
mated number of background events to the total number of events in the response spectrum. These contributions are summarized in Table III.

The calorimeter resolution was calculated by dividing the measured rms at each energy by the corresponding mean. The beam energy spread [14] given in table II and scraping corrections given in table III were then subtracted in quadrature. The corrected calorimeter resolution $\left(\sigma_{E} / E\right)$ was then plotted as a function of beam energy. Plots of the energy resolution as a function of incident electron energy are shown for the diamond and silicon calorimeters in Figure 10. The smooth curves are fits to functions of the form:

$$
\frac{\sigma_{E}}{E}=\sqrt{\frac{A^{2}}{E^{2}}+\frac{B^{2}}{E}+C^{2}} \equiv \frac{A}{E} \oplus \frac{B}{\sqrt{E}} \oplus C
$$

The values for the fit parameters along with their $\chi^{2}$ are summarized in Table IV. The fit to the diamond data accidentally gives a small $\chi^{2}$. The fit parameters were found to be highly correlated as evidenced from the correlation matrices in Table V.

\section{CALORIMETER SIMULATION}

A simulation of each calorimeter using EGS-IV [15] was performed to compare with the measured resolutions and to determine the amount of lateral and longitudinal energy loss. Because the detector elements were very thin, the EGS results were sensitive to the minimum cutoff energies used. Therefore, these values were set very low at $0.1 \mathrm{MeV}$ kinetic energy for both electrons and photons. Since, in the analysis, events were accepted where the incident beam struck a $1.0 \times 1.0 \mathrm{~cm}^{2}$ square centered in the calorimeter, the simulation generated events where the incident electrons were uniformly populated over this region. Because of the high statistics in our two data samples, similarly large samples of EGS events were required. The simulations presented here represent approximately 700 cpu-hours on 100 Mips computers.

The resolutions given by the EGS simulation are shown in Figure 11 and in Table IV. The lateral energy leakage was $18 \%$, independent of the beam energy while the fluctuations on the lateral leakage varied approximately as $3.4 \% / \sqrt{E}$. The longitudinal energy leakage depended on the beam energy reaching $1.4 \%$ at $5 \mathrm{GeV}$. The fluctuations on the longitudinal leakage were $1.1 \%$, independent of the beam energy.

\section{DISCUSSION}

The resolution of calorimeters are usually quoted in terms of the last two parameters of equation 4.1 [16]. However, because of the high statistics and relatively low energies measured, the two parameter fits were insufficient to describe our data. The three parameter fits used include terms which vary as $1 / E, 1 / \sqrt{E}$ and a constant. In particular, the $1 / E$ dependence was found to be important at low energy.

The $1 / E$ term arises from effects which produce a constant width to the calorimeter response $\left(\sigma_{E}\right)$. The simulations of both calorimeters indicate a $2-3 \% / E$ dependence. This is attributed to the high energy tail of the Landau distribution for single tracks [13]. An additional contribution arises from electronic noise in each of the detector channels. By adding 
the single channel noise for all the channels in quadrature, we estimate the contribution to the $1 / E$ term due to electronic noise for each calorimeter. Compared with the results from the EGS simulations, the noise contribution in the diamond calorimeter is large, $(7 \pm 1) \% / E$ whereas in the silicon calorimeter this contribution is small, $(0.9 \pm 0.1) \% / E$. If the noise contributions to the calorimeter resolutions are subtracted in quadrature and the data refit, the $1 / E$ dependence for both diamond and silicon are $(4.7 \pm 2.7 \%) / E$ and $(3.89 \pm 0.87 \% / E)$, respectively, while the other terms remain unchanged. This brings the diamond and silicon $1 / E$ dependence into agreement with each other and with the EGS simulations.

The $1 / \sqrt{E}$ dependence arises from counting statistics on the number of particles passing through the detector layers. As noted above, the lateral leakage also contributes to this term. However, the contribution of $3.4 \% / \sqrt{E}$ to the overall resolution is negligible when compared with the counting statistics of the contained portion of the shower. For both detectors, we find approximately a $19 \% / \sqrt{E}$ term in both the data and simulation, in substantial agreement with the EGS results.

Since only $82 \%$ of the shower is contained, on average, the numbers above are not the intrinsic resolutions with $1 X_{0}$ sampling. If the showers were fully contained, the resolutions would be $\sqrt{0.82}$ smaller than above. Our results, therefore correspond to an intrinsic $1 / \sqrt{E}$ dependence for $1 X_{0}$ diamond-tungsten and $1 X_{0}$ silicon-tungsten sampling of $17.32 \pm 0.77 \% / \sqrt{E}$ and $17.87 \pm 0.17 \% / \sqrt{E}$, respectively.

The constant term arises from sources whose contributions to the width of the energy distributions vary linearly with the energy. From the simulations, fluctuations in the longitudinal leakage are seen to contribute approximately 1.1\%. Both fits to the data yield results consistent with this number.

\section{SUMMARY OF RESULTS}

We have constructed and successfully operated simple diamond-tungsten and silicontungsten calorimeters with $1.0 X_{0}$ sampling frequency. Our results from exposure of these calorimeters to electron beams ranging between $0.5-5.0 \mathrm{GeV}$ show that both detectors have a linear response in beam energy while their energy resolutions are comparable. The energy resolution of the both calorimeters, subtracting contributions due to electronic noise of $(7 \pm 1) \% / E$ for diamond and $(0.9 \pm 0.1) \% / E$ for silicon were found to be:

$$
\begin{aligned}
& \text { Diamond: } \quad \frac{\sigma_{E}}{E}=\frac{(4.7 \pm 2.7) \%}{E} \oplus \frac{(19.13 \pm 0.86) \%}{\sqrt{E}} \oplus(2.3 \pm 1.8) \% \\
& \text { Silicon: } \quad \frac{\sigma_{E}}{E}=\frac{(4.00 \pm 0.85) \%}{E} \oplus \frac{(19.73 \pm 0.19) \%}{\sqrt{E}} \oplus(0.0 \pm 1.6) \%
\end{aligned}
$$

respectively. These results show good agreement between the diamond and silicon calorimeters. Reasonable agreement is also found between the data and EGS simulations of the calorimeters.

We gratefully acknowledge the Texas National Research Laboratory Commission and the Superconducting Supercollider Laboratory for sponsoring this research. We are particularly grateful to W. Chinowsky, F. Gilman and R. Schwitters for their encouragement and support 
of this project. K. K. Gan and S. K. Kim thank the TNRLC for sponsoring the SSC fellowship program. S. K. Kim also acknowledges support from the S.N.U. Daewoo Research Fund. 


\section{REFERENCES}

[1] L.S. Pan, et al., "Journal of Applied Physics," 74 (1993) 1086.

[2] Norton Diamond Film, Northboro, MA 01532-1545.

[3] S. Zhao, Ph.D. Thesis, Ohio State University,1994 (unpublished).

[4] Fujikura Kasei Co., Ltd., Sakae-machi, Sano-city, Tochigi-ken, Japan.

[5] M. Franklin, et al., "Nuclear Instruments and Methods," A315 (1992) 39.

[6] Hamamatsu Corporation, Bridgewater, NJ. 08807-0910.

[7] T. Tanaguchi, Y. Fukushima, Y. Yoribayashi, "IEEE Transactions on Nuclear Science" NS-36 (1989) 657.

[8] P. Wilson, PhD. Thesis, Ohio State University (1992).; Y. Kubota, et al., "Nuclear Instruments and Methods," A320 (1992) 66.

[9] LeCroy Research Corporation, Chesnut Ridge, NY 10977.

[10] J. Anderson, J. Franzen, Data Acquisition Group, Fermi National Accelerator Laboratory, Batavia, IL 60510.

[11] Particle Data Group "Physical Review D" 45 (1992) III-14.

[12] Digital Equipment Corporation, Westminister, MA 01473-0471.

[13] H. G. Fischer, "Nuclear Instruments and Methods," 156 (1978) 81.

[14] K. Ogawa, et al., KEK internal memo 85-2.

[15] R. L. Ford and W. R. Nelson, SLAC-0210 (1978).

[16] S. Almehed, et al., "Nuclear Instruments and Methods," A305 (1991) 320; J. Bourotte, et al., "Nuclear Instruments and Methods," A274 (1989) 129; G. Barbiellini, et al., "Nuclear Instruments and Methods," A 257 (1986) 543; A. Nakamoto, et al., "Nuclear Instruments and Methods," A251 (1986) 275; M. Bormann, et al., "Nuclear Instruments and Methods," A240 (1985) 63; A. Nakamoto, et al., "Nuclear Instruments and Methods," A238 (1985) 53; G. Barbiellini, et al., "Nuclear Instruments and Methods," A235 (1985) 55. 


\section{TABLES}

TABLE I. Diamond parameters and location in the calorimeter

\begin{tabular}{rccc}
\hline \hline $\begin{array}{c}\text { Detector } \\
\text { Layer }\end{array}$ & $\begin{array}{c}\text { Diamond } \\
\text { Thickness }(\mu \mathrm{m})\end{array}$ & $\begin{array}{c}\text { Collection } \\
\text { Distance }(\mu \mathrm{m})\end{array}$ & $\begin{array}{c}\text { Operating } \\
\text { Voltage }(\mathrm{V})\end{array}$ \\
\hline 1 & 630 & 40 & 300 \\
2 & 610 & 46 & 300 \\
3 & 534 & 44 & 500 \\
4 & 562 & 50 & 600 \\
5 & 359 & 38 & 400 \\
6 & 328 & 36 & 300 \\
7 & 360 & 38 & 400 \\
8 & 500 & 35 & 400 \\
9 & 521 & 38 & 500 \\
10 & 470 & 44 & 500 \\
11 & 497 & 42 & 500 \\
12 & 499 & 44 & 500 \\
13 & 584 & 48 & 250 \\
14 & 547 & 44 & 500 \\
15 & 564 & 46 & 500 \\
16 & 206 & 36 & 150 \\
17 & 587 & 46 & 200 \\
18 & 643 & 35 & 100 \\
19 & 564 & 44 & 500 \\
20 & 591 & 36 & 100 \\
\hline Average & 507 & 42 & - \\
\hline \hline
\end{tabular}

TABLE II. Electron beam energies and calorimeter responses for both silicon and diamond calorimeters.

\begin{tabular}{cccccc}
\hline \hline \multicolumn{2}{c}{ Beam Characteristics } & \multicolumn{2}{c}{ Diamond Calorimeter } & \multicolumn{2}{c}{ Silicon Calorimeter } \\
$P(\mathrm{GeV})$ & $\sigma_{P} / P(\%)[14]$ & $<E>(\mathrm{MIP}$ 's $)$ & $E_{R M S}$ (MIP's) & $<E>(\mathrm{MIP}$ 's) & $E_{R M S}$ (MIP's) \\
\hline 0.5 & $4.9 \pm 0.37$ & $32.09 \pm 0.13$ & $10.38 \pm 0.10$ & $32.33 \pm 0.12$ & $9.49 \pm 0.09$ \\
1.0 & $4.0 \pm 0.30$ & $66.15 \pm 0.15$ & $14.24 \pm 0.10$ & $65.31 \pm 0.13$ & $13.62 \pm 0.09$ \\
2.0 & $2.8 \pm 0.21$ & $133.40 \pm 0.18$ & $19.73 \pm 0.13$ & $132.60 \pm 0.17$ & $19.30 \pm 0.12$ \\
3.0 & $2.2 \pm 0.17$ & $201.50 \pm 0.22$ & $24.37 \pm 0.15$ & $200.30 \pm 0.22$ & $23.97 \pm 0.16$ \\
4.0 & $1.6 \pm 0.12$ & $264.80 \pm 0.34$ & $27.94 \pm 0.24$ & $268.10 \pm 0.35$ & $28.19 \pm 0.25$ \\
5.0 & $1.3 \pm 0.10$ & $331.80 \pm 0.34$ & $32.01 \pm 0.24$ & $328.50 \pm 0.38$ & $30.58 \pm 0.27$ \\
\hline \hline
\end{tabular}


TABLE III. Beam scraping corrections to the resolutions of the diamond and silicon calorimeters.

\begin{tabular}{ccc}
\hline \hline & Diamond Calorimeter & Silicon Calorimeter \\
Beam Energy $(\mathrm{GeV})$ & $\sigma_{E} / E(\%)$ & $\sigma_{E} / E(\%)$ \\
\hline 0.5 & $1.75 \pm 0.41$ & $2.10 \pm 0.46$ \\
1.0 & $1.90 \pm 0.42$ & $2.60 \pm 0.56$ \\
2.0 & $2.20 \pm 0.48$ & $2.40 \pm 0.52$ \\
3.0 & $2.50 \pm 0.54$ & $2.90 \pm 0.61$ \\
4.0 & $2.80 \pm 0.59$ & $3.10 \pm 0.65$ \\
5.0 & $3.10 \pm 0.65$ & $3.30 \pm 0.68$ \\
\hline \hline
\end{tabular}

TABLE IV. Fitted resolutions for silicon and diamond calorimeters.

\begin{tabular}{ccccc}
\hline \hline Detector & $A(\% \mathrm{GeV})$ & $B\left(\% G e V^{\frac{1}{2}}\right)$ & $C(\%)$ & $\chi^{2} /$ D.O.F. \\
\hline Diamond(data) & $8.35 \pm 0.73$ & $19.20 \pm 0.52$ & $2.2 \pm 1.3$ & $0.04 / 3$ \\
Diamond(EGS) & $2.94 \pm 0.56$ & $18.62 \pm 0.15$ & $1.33 \pm 0.49$ & $3.26 / 3$ \\
Silicon(data) & $4.00 \pm 0.85$ & $19.73 \pm 0.19$ & $0.0 \pm 1.6$ & $2.21 / 3$ \\
Silicon(EGS) & $1.6 \pm 0.67$ & $20.578 \pm 0.047$ & $0.00 \pm 0.91$ & $2.82 / 3$ \\
\hline \hline
\end{tabular}

TABLE V. Correlation matrices for the fits to the silicon and diamond calorimeter data.

\begin{tabular}{c|ccc|ccc|}
\hline \hline & Silicon & & \multicolumn{3}{c|}{ Diamond } \\
& $A$ & $B$ & $C$ & $A$ & $B$ & $C$ \\
\hline$A$ & 1.000 & -0.946 & 0.804 & 1.000 & -0.952 & 0.803 \\
$B$ & -0.946 & 1.000 & -0.912 & -0.952 & 1.000 & -0.908 \\
$C$ & 0.804 & -0.912 & 1.000 & 0.803 & -0.908 & 1.000 \\
\hline \hline
\end{tabular}


FIG. 1. Schematic of diamond detector operation. 
FIG. 2. Diamond detector assembly. The ground pad is connected through the side of the ceramic board opposite that shown. The electrode structure of a diamond wafer is shown in the lower diagram. Thicknesses of the electrodes are greatly exaggerated for clarity. 


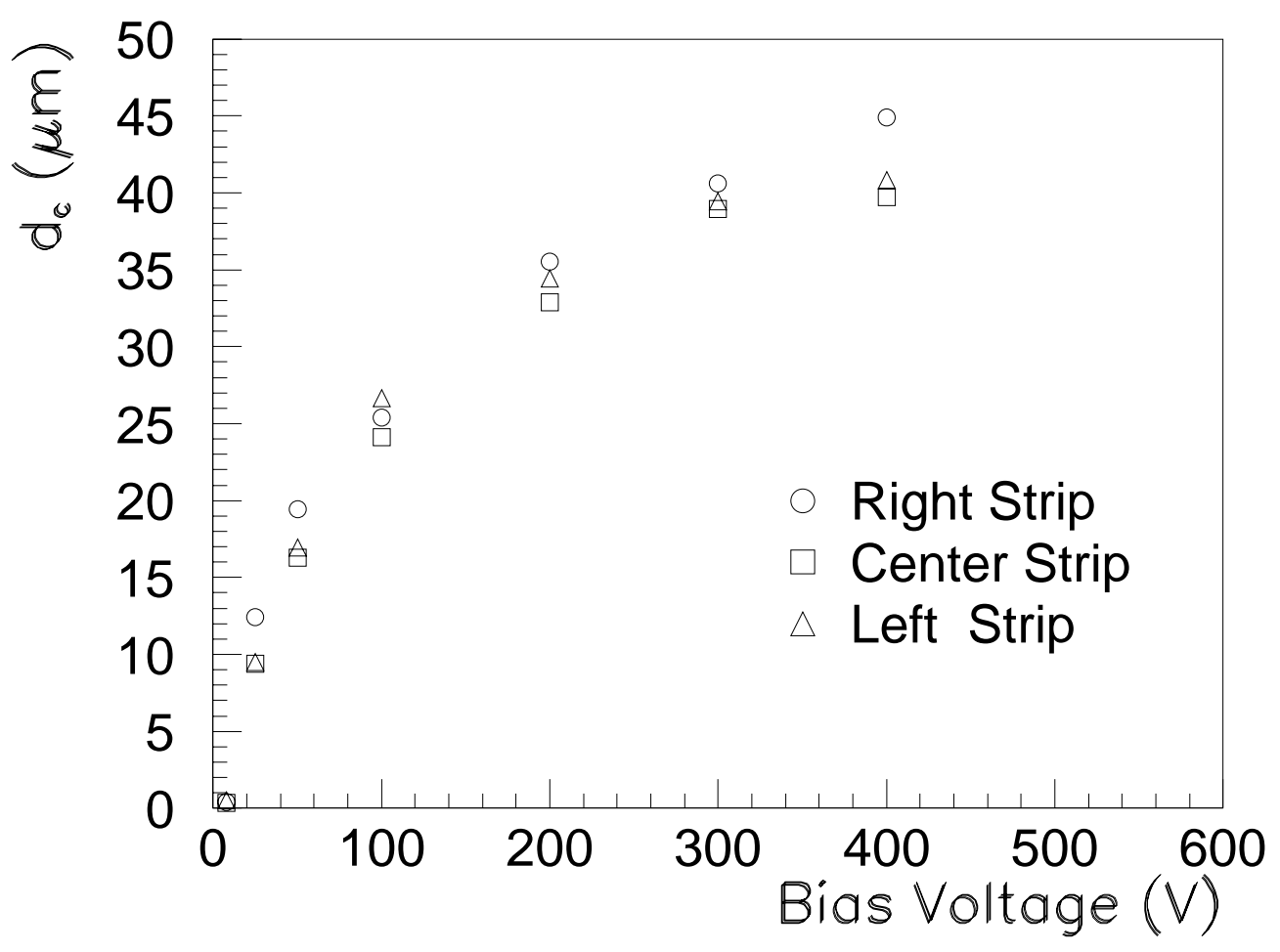

FIG. 3. Typical diamond collection distance $\left(d_{c}\right)$ as a function of bias voltage. All three strips are shown on the graph. 
FIG. 4. Schematic of the calorimeter electronics. Differences between the diamond and silicon setup are noted in the diagram. 
FIG. 5. Schematic diagram of calorimeter data acquisition system. 


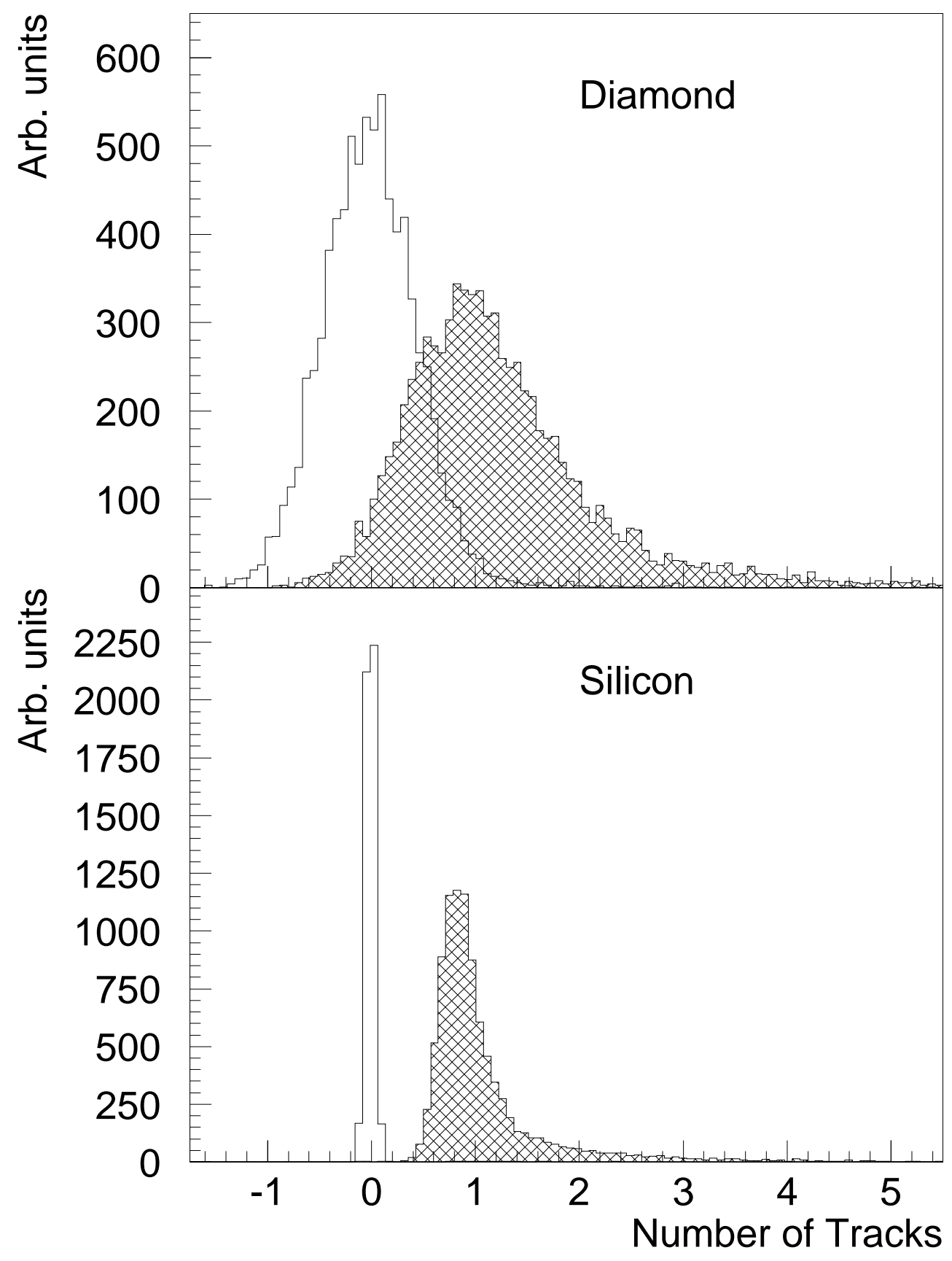

FIG. 6. Single particle signals for individual diamond and silicon detectors (shaded regions). The unshaded regions are noise signals from empty events. 


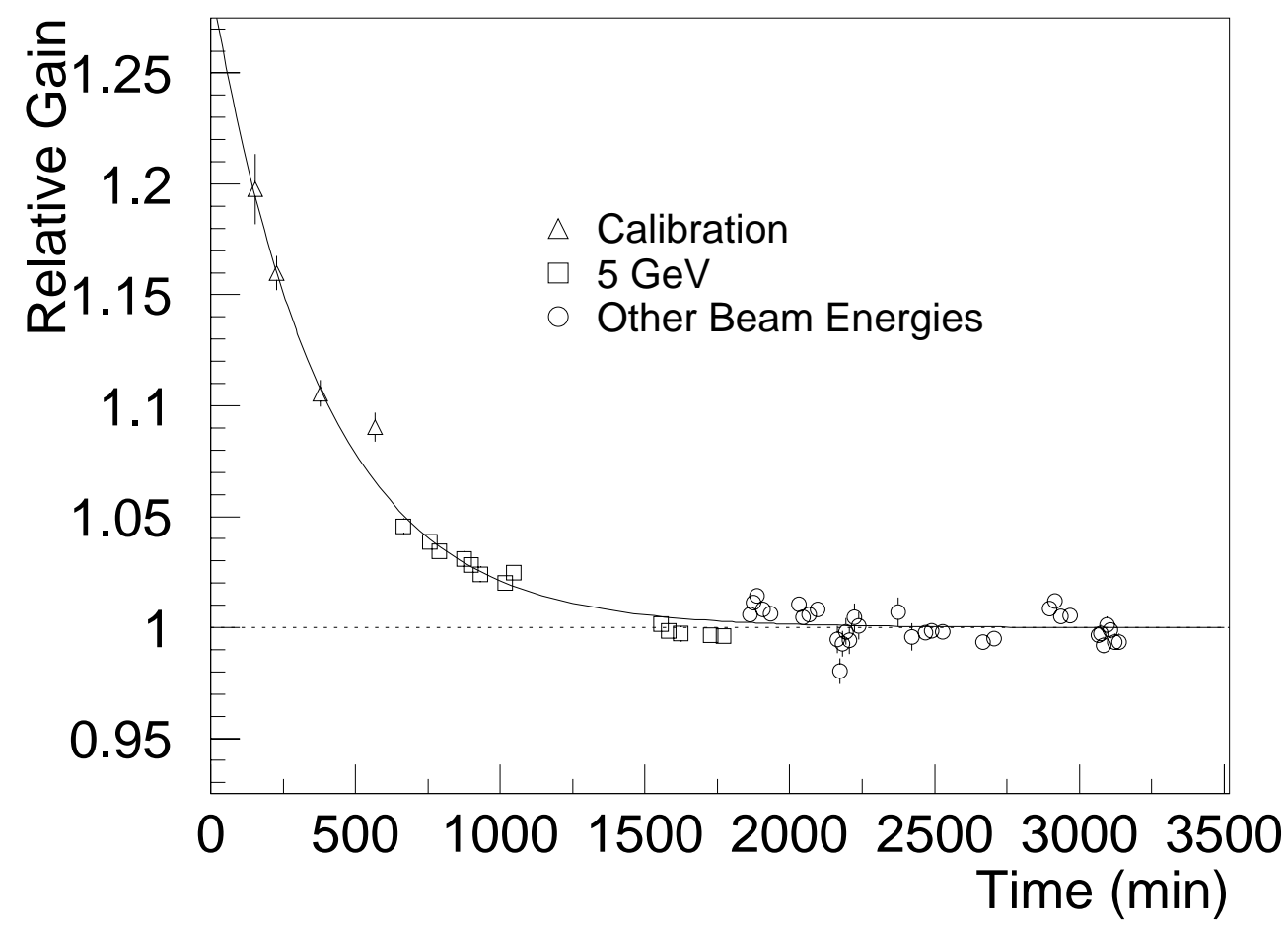

FIG. 7. Relative calorimeter gain as a function of time since the application of the bias voltage. The solid line corresponds to the fit discussed in the text. 


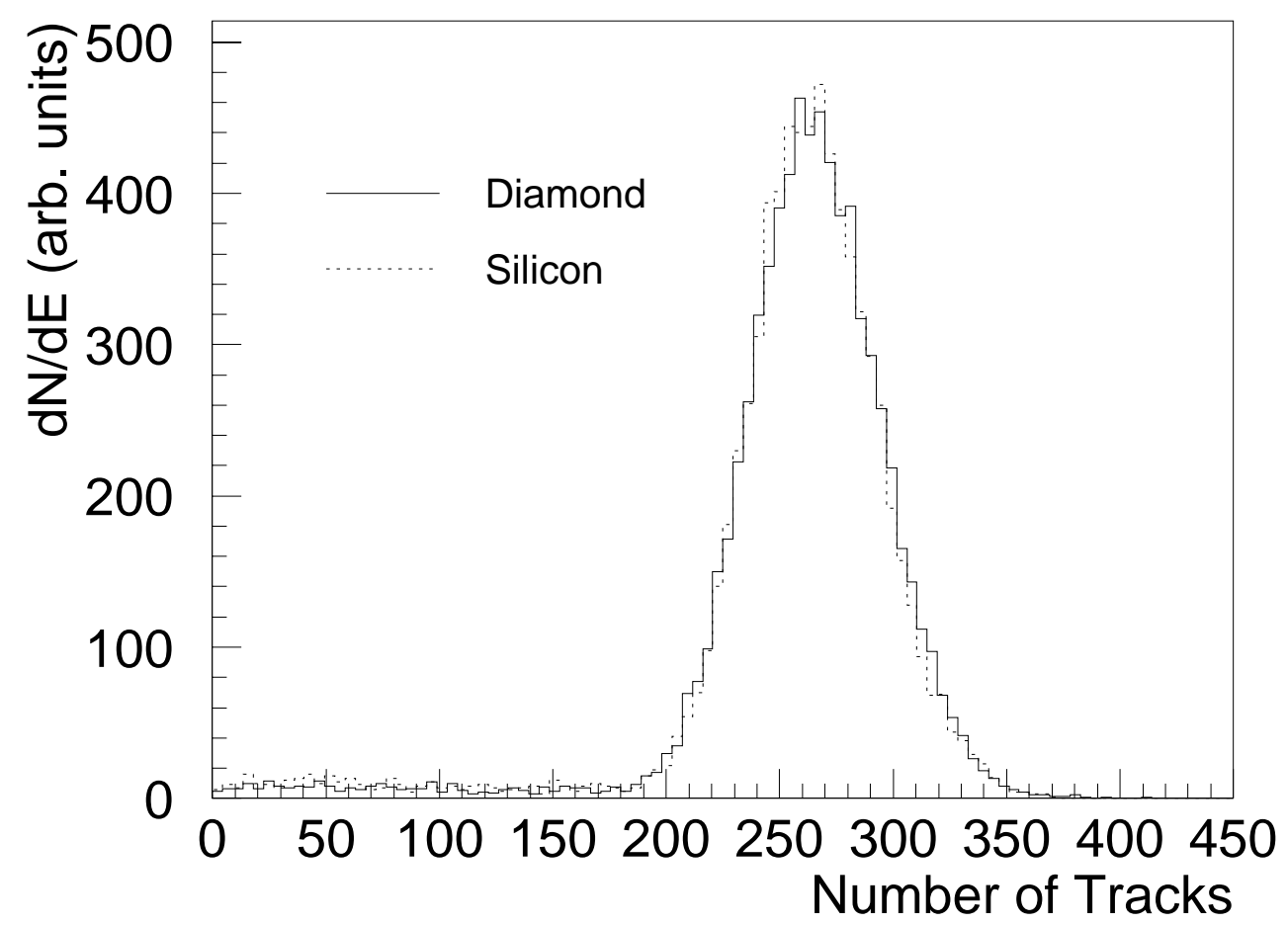

FIG. 8. Calorimeter response to $4 \mathrm{GeV}$ electrons for both calorimeters. 


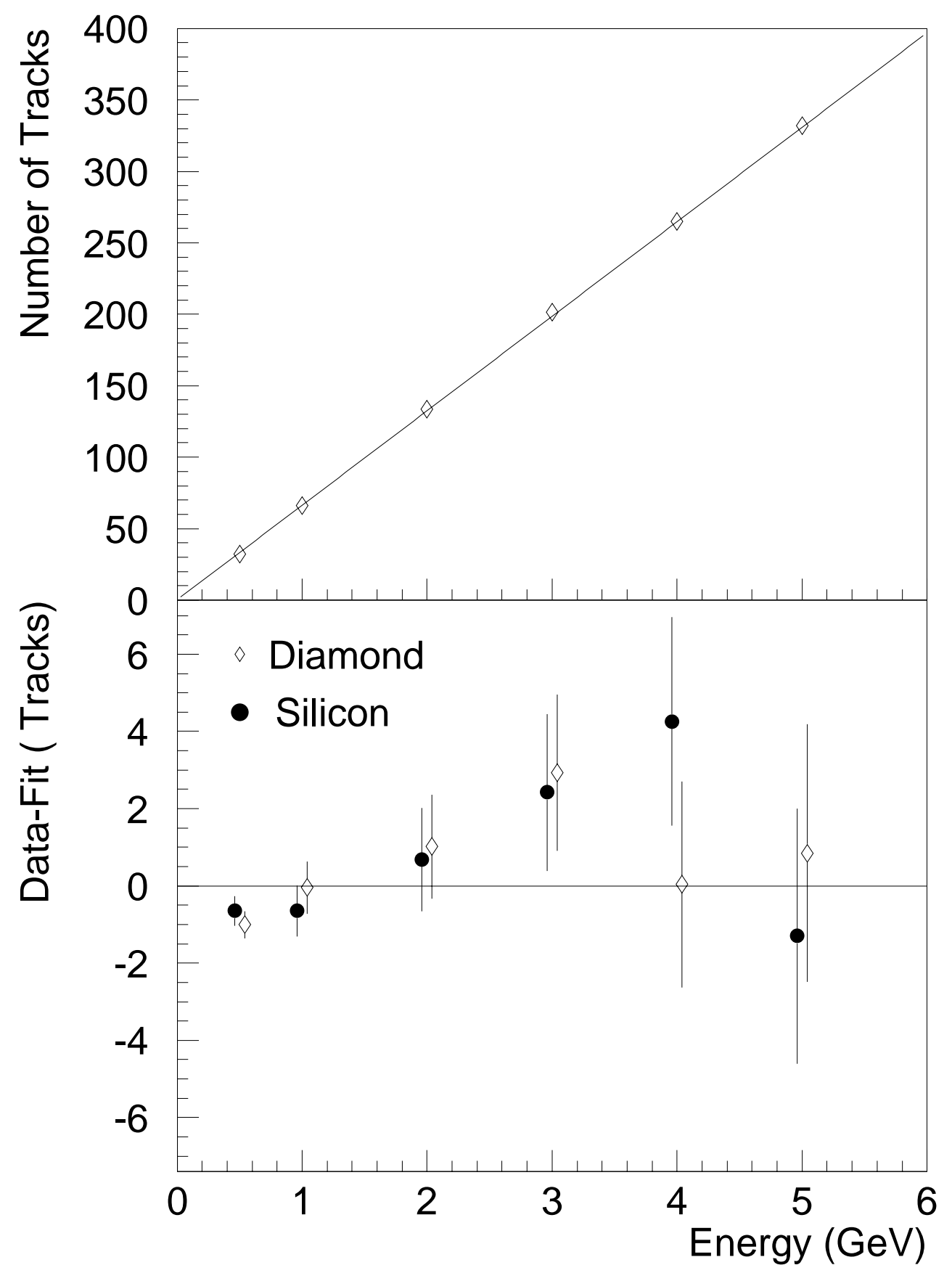

FIG. 9. Calorimeter response as a function of electron beam momentum. The lower graph shows the difference between the data and a linear fit to the data. 


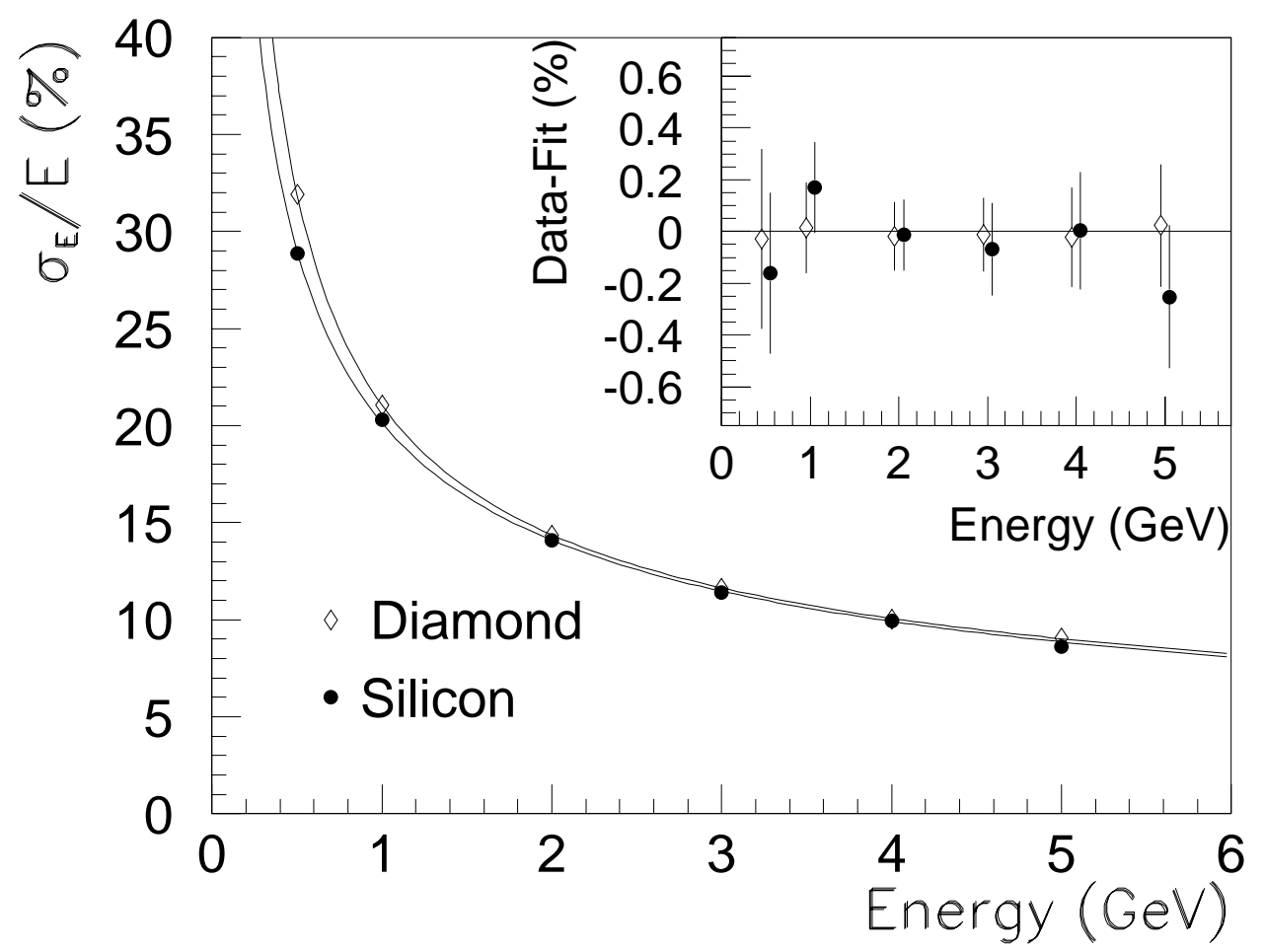

FIG. 10. Energy resolution ( $\left.\sigma_{E} / E\right)$ of a) silicon and b) diamond based calorimeters as a function of energy. The inset shows the residuals for the fits discussed in the text. 


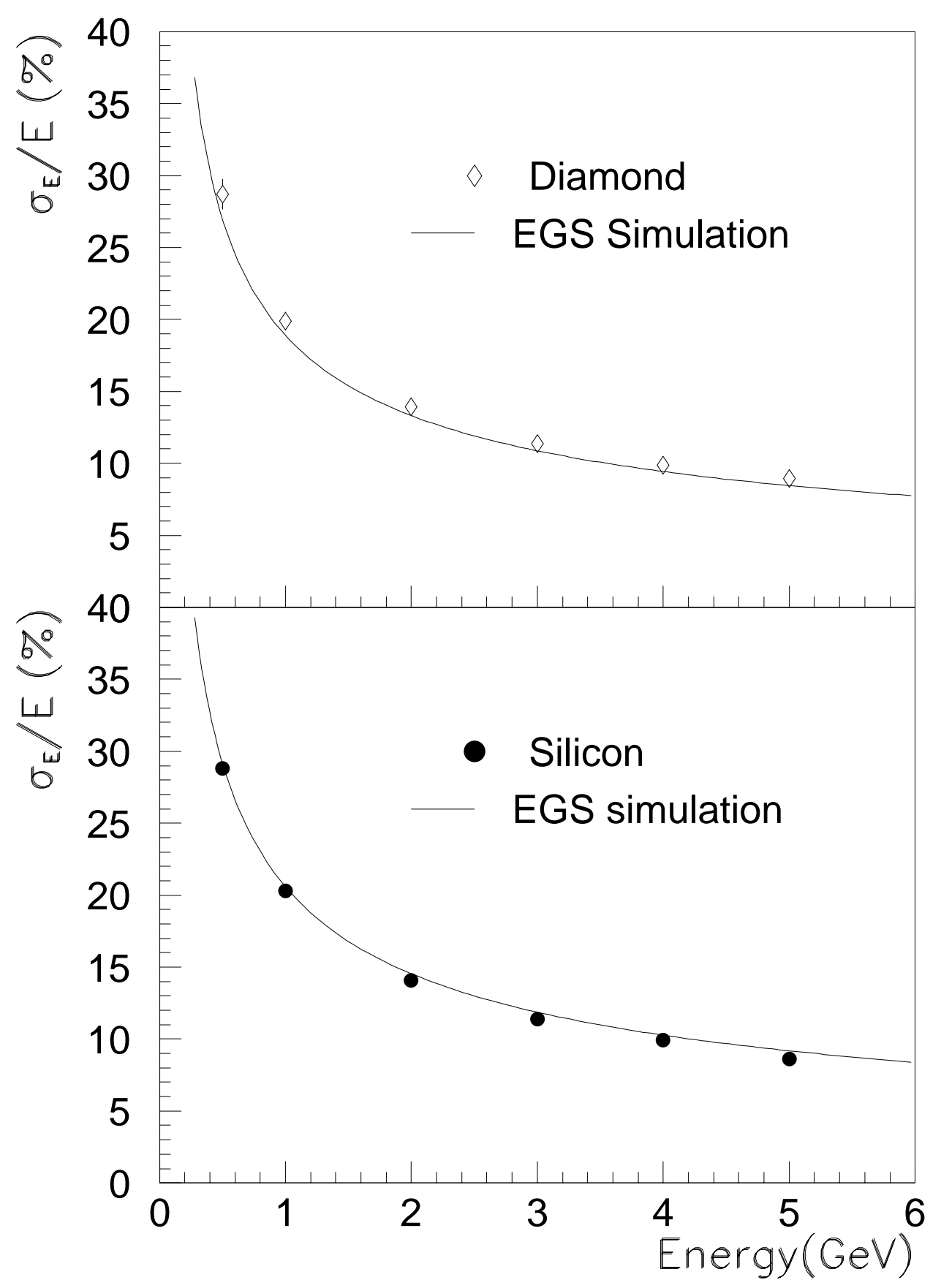

FIG. 11. Comparison of the energy resolution $\left(\sigma_{E} / E\right)$ of diamond and silicon calorimeters with EGS simulations. The data from both calorimeters have electronic noise contributions to the resolution subtracted in quadrature. 